Pacific Journal of Mathematic 


\title{
ON THE EXTREMAL ELEMENTS OF THE CONVEX CONE OF SUPERADDITIVE $n$-HOMOGENEOUS FUNCTIONS
}

\author{
Melvyn W. Jeter
}

Let $P_{n}$ be the collection of finite-valued functions defined on the nonnegative orthant, $E_{n^{2}}^{+}$, of euclidean $n^{2}$-space such that for $p \in P_{n}$ it follows that $p: E_{n}^{+}{ }^{2} \rightarrow E_{1}^{+}$and in addition
(a) $p$ is continuous,
(b) $p(\alpha x)=\alpha^{n} p(x), \alpha \geqq 0$,
(c) $p(x+y) \geqq p(x)+p(y)$.

It follows readily that $P_{n}$ is closed with respect to addition and nonnegative scalar multiplication. Therefore, $P_{n}$ is a convex cone, whose vertex is the zero function, in the linear space of real functions defined on $E_{n^{2}}^{+}$. The purpose of this paper is to investigate the extremal elements of $P_{n}$.

1. Introduction. One well known member of $P_{n}$ is the permanent function. Recently functions that generalize the permanent function have been studied by Rothaus [9] and new representations for the permanent function have been sought (for example see [2] by Marcus and Newman). The interest in determining the extremal elements of $P_{n}$ comes from the fact that under certain circumstances it is possible to give an integral representation for any $p \in P_{n}$ in terms of the extremal elements of $P_{n}$ [1] (examples of similar studies may be found in papers by McLachlan [4], [5], [6] and Rakestraw [7]). In this paper it is shown that for $a \in E_{n^{2}}^{+} \backslash 0$, the functions $p_{a}(x)=$ $\sup \left\{\lambda^{n}: x \geqq \lambda a\right\}$ are extremal elements of $P_{n}$. Replacing condition (b) by

(b) $p(\alpha x)=\alpha p(x), \alpha \geqq 0$,

gives the collection of monotone concave gauges, denoted by $P_{n}^{\prime}$, defined on $E_{n^{2}}^{+}$[8]. If for all $i=1, \cdots, n, A_{i} \in P_{n}^{\prime}$, then the function $A$ defined as $A(x)=\prod_{i=1}^{n} A_{i}(x)$ is an element of $P_{n}$. If $S_{n}$ denotes all those $p \in P_{n}$ which are finite nonnegative linear combination of functions of this type, then clearly $S_{n}$ is a subcone of $P_{n}$ and $S_{n}$ contains the permanent function. It is shown here that for a function $p \in S_{n}$ to be an extremal element of $P_{n}$, then $p$ must be of the form $p(x)=[A(x)]^{n}$, where $A(x)$ is an extremal element of $P_{n}^{\prime}$. 
In the material to follow define $[p: \alpha]=\{x: p(x)=\alpha\}$, where $p \in P_{n}$. It follows that $\alpha[p: 1]=\left[p: \alpha^{n}\right]$ for all $\alpha \geqq 0$. Also, use will be made of the fact that for $p \in P_{n}^{\prime}$ or $p \in P_{n}$, then $x \geqq y$ (or $x>y$ ) implies that $p(x) \geqq p(y)$ (or $p(x)>p(y)$ ). Further if $x \in$ int $E_{n^{2}}^{+}$and $p \neq 0$, then $p(x)>0$.

2. Extremal elements of $P_{n}$. The first theorem of this section gives some of the extremal elements of $P_{n}$. It is conjectured that this set includes all the extremal elements of $P_{n}$. The following lemmas will be needed.

Lemma 1.1. If $p, q \in P_{n}$. Define

$$
(p \wedge q)(x)=\min \{p(x), q(x)\} .
$$

Then $p \wedge q \in P_{n}$.

Proof. It follows readily from the definitions that $p \wedge q$ is continuous and homogeneous. Also,

$$
\begin{aligned}
(p \wedge q)(x+y) & =\min \{p(x+y), q(x+y)\} \\
& \geqq \min \{p(x)+p(y), q(x)+q(y)\} \\
& \geqq \min \{p(x), q(x)\}+\min \{p(y), q(y)\} \\
& =(p \wedge q)(x)+(p \wedge q)(y) .
\end{aligned}
$$

For all $k=1, \cdots, n^{2}$, let $p_{h}(x)=x_{h}^{n}, x=\left(x_{1}, \cdots, x_{n^{2}}\right) \in E_{n^{2}}^{+}$. Then $p_{k} \in P_{n}$. With this in mind consider the following:

Lemma 1.2. Let $a=\left(a_{1}, \cdots, a_{n^{2}}\right) \in E_{n^{2}}^{+} \backslash\{0\}$. Define $p_{a}$ as follows:

$$
p_{a}(x)=\sup \left\{\lambda^{n}: x \geqq \lambda a, \lambda \geqq 0\right\} .
$$

Then $p_{a} \in P_{n}$.

Proof. Without loss of generality, assume the nonzero coordinates of $a$ are $a_{1}, \cdots, a_{k}, k \leqq n^{2}$. Let

$$
p(x)=\left(\frac{1}{a_{1}^{n}} p_{1} \wedge \cdots \wedge \frac{1}{a_{h}^{n}} p_{h}\right)(x) .
$$

Lemma 1.1 implies that $p \in P_{n}$. Now for any given $x \in E_{n^{2}}^{+}$suppose that 


$$
p(x)=\frac{x_{l}^{n}}{a_{l}^{n}}
$$

$1 \leqq l \leqq k$. Then it follows readily that for each $i$

$$
x_{i} \geqq \frac{x_{l}}{a_{l}} a_{i}
$$

with equality when $i=l$. Further there does not exist $\lambda>x_{l} / a_{l}$ such that $x \geqq \lambda a$ since otherwise

$$
x_{l}>\lambda a_{l}>\frac{x_{l}}{a_{l}} a_{l}=x_{l}
$$

Hence, $p_{a}(x)=p(x)$ for every $x \in E_{n^{2}}^{+}$, which implies that $p_{a}=p$.

Notice that if $a_{i}$ is a nonzero coordinate of $a$ and $x \in E_{n^{2}}^{+}$such that $x_{i}=0$, then $x \geqq \lambda a$ implies that $\lambda=0$. Thus, $p_{a}(x)=0$. Also, if $a=e_{k}$, where $e_{k}$ is that vector having all zero coordinates except the $k$ th coordinate which is 1 , then $p_{a}=p_{k}$.

In general, if $p \in P_{n}$ the set [ $\left.p: 1\right]$ is difficult to characterize. For example a complete characterization of the set $\left[p: n ! / n^{n}\right]$ (and hence $[p: 1])$ where $p$ is the permanent function is not known [3]. However, if $p=p_{a}$ for some $a \neq 0$, then a characterization is possible. Let $a=\left(a_{1}, \cdots, a_{n^{2}}\right) \in E_{n^{2}}^{+}, a \neq 0$. For every $i \in\left\{1, \cdots n^{2}\right\}$, define

$$
R\left(a_{i}\right)=\left\{\left(x_{1}, \cdots, x_{i-1}, a_{i}, x_{i+1}, \cdots, x_{n^{2}}\right): x_{j} \geqq a_{j} \text { for } j \neq i\right\} .
$$

Lemma 1.3. If $a \in E_{n^{2}}^{+} \backslash\{0\}$, then

$$
\left[p_{a}: 1\right]=\cup\left\{R\left(a_{i}\right): a_{i} \neq 0\right\}
$$

Proof. Let $y \in R\left(a_{i}\right)$, where $a_{i} \neq 0$. Clearly, $y \geqq a$. Notice there does not exist $\lambda>1$ such that $y \geqq \lambda a$, for otherwise $a_{i} \geqq \lambda a_{i}>$ $a_{i}$. Hence, by definition $p_{a}(y)=1$. This implies that

$$
\cup\left\{R\left(a_{i}\right): a_{i} \neq 0\right\} \subset\left[p_{a}: 1\right] \text {. }
$$

Now suppose $y \in\left[p_{a}: 1\right]$. Considering (1.1), there exists $k \in$ $\left\{1, \cdots, n^{2}\right\}$ such that $a_{k}>0$ and $\left(y_{k} / a_{k}\right)^{n}=1$. This implies that $y_{k}^{n}=a_{k}^{n}$, which implies that $y_{k}=a_{k}$. For all other $i \in\left\{1, \cdots, n^{2}\right\}$ such that $a_{i}>0$, $\left(y_{i} / a_{i}\right)^{n} \geqq 1$ and hence $y_{i} \geqq a_{i}$. It follows that $y \in R\left(a_{k}\right)$ and the proof is complete.

Using this result it is possible to show that $p_{a}=p_{b}$ if and only if $a=b$. Next using Lemma 1.3, $p_{a}$ is shown to be an extremal element of $P_{n}$. 
THEOREM 1.1. The function $p_{a}$ is an extremal element of $P_{n}$.

Proof. Suppose $p_{a}=f+g$. Let $y \in R\left(a_{i}\right)$, where $a_{i} \neq 0$ and $i \in$ $\left\{1, \cdots, n^{2}\right\}$, then

$$
P_{a}(a)=P_{a}(y)=f(y)+g(y) \geqq f(a)+g(a)=P_{a}(a) .
$$

This implies $f(y)=f(a)$ and $g(y)=g(a)$, since $f(y) \geqq f(a)$ and $g(y) \geqq$ $g(a)$. Also, $p_{a}(a)=f(a)+g(a)$ implies $p_{a}(a) \geqq f(a)$ and $p_{a}(a) \geqq$ $g(a)$. Therefore, there exists $\alpha \geqq 0$ and $\beta \geqq 0$ such that $\alpha p_{a}(a)=f(a)$ and $\beta p_{a}(a)=g(a)$.

Again, without loss of generality, suppose the nonzero coordinates of $a$ are $a_{1}, \cdots, a_{k}$. Let $x \in E_{n^{2}}^{+}$such that $x_{1}>0, \cdots, x_{k}>0$. Then for every $i \in\{1, \cdots, k\}$ there exists $\lambda_{i}>0$ such that $a_{i}=\lambda_{i} x_{i}$. Let $\lambda=$ $\max \left\{\lambda_{i}: i \in\{1, \cdots, k\}\right\}$. Notice there exists a $j \in\{1, \cdots, k\}$ such that $\lambda=\lambda_{j}$. Hence, $\lambda x_{i} \geqq a_{i}$ with equality when $i=j$. Clearly, if $i \in\left\{1, \cdots, n^{2}\right\} \backslash\{1, \cdots k\}$, then $\lambda x_{i} \geqq a_{i}$. Therefore, $\lambda x \in R\left(a_{j}\right)$. Setting $y=\lambda x$, it follows that

$$
\begin{aligned}
f(x) & =f\left(\frac{1}{\lambda} y\right)=\frac{1}{\lambda^{n}} f(y) \\
& =\frac{1}{\lambda^{n}} f(a)=\frac{1}{\lambda_{n}} \alpha p_{a}(a) \\
& =\frac{1}{\lambda^{n}} \alpha p_{a}(y)=\alpha p_{a}\left(\frac{1}{\lambda} y\right) \\
& =\alpha p_{a}(x) .
\end{aligned}
$$

Clearly, if $x \in E_{n^{2}}^{+}$such that $x_{i}=0$ for some $i \in\{1, \cdots, k\}$, then $0=$ $p_{a}(x)$. This implies $f(x)=0$, which in turn implies that $f(x)=$ $\alpha p_{a}(x)$. In either case $f(x)=\alpha p_{a}(x)$. Hence, $f=\alpha p_{a}$. Likewise, $g=$ $\beta p_{a}$. Therefore, $p_{a}$ is an extremal element of $P_{n}$.

By a somewhat similar proof it can be shown that the function $p_{a}$ is minimal in the set of all elements of $P_{n}$ which agree with $p_{a}(a)$ at a. Also, for $\alpha>0$ the sets $\left[p_{a}: 1\right]$ have the property that $\alpha\left[p_{a}: 1\right]=$ $\left[p_{a}: \alpha^{n}\right]=\left[p_{\alpha a}: 1\right]$.

Recall that $S_{n} \subset P_{n}$ is the set of finite nonnegative linear combinations of products of $n$ functions of $P_{n}^{\prime}$. For $a \in E_{n^{2}}^{+} \backslash\{0\}$, let $q_{a}(x)=$ $\sup \{\lambda: x \geqq \lambda a\}$. Then, as in the case for $P_{n}, q_{a}$ is an extremal element of $P_{n}^{\prime}$. Also, $p_{a}(x)=\left[q_{a}(x)\right]^{n}$, which implies that $p_{a} \in S_{n}$. Since $S_{n}$ is a subcone of $P_{n}$ then $p_{a}$ is an extremal element of $S_{n}$. It is conjectured that $\left\{p_{a}: a \in E_{n^{2}}^{+}\{0\}\right\}$ represents all the extremal elements of $S_{n}$. 
Lemma 1.4. If $p \neq 0$ and $p(x)=\prod_{i=1}^{n} A_{i}(x)$, where $A_{i} \in P_{n}^{\prime}$, is an extremal element of $S_{n}$, then each $A_{i}$ is an extremal element of $P_{n}^{\prime}$.

Proof. Suppose there exists a $k=1, \cdots, n$ such that $A_{k}$ is not extremal in $P_{n}^{\prime}$. Then there exists $f, g \in P_{n}^{\prime}$ such that $A_{k}=f+g$ and neither $f$ or $g$ is proportional to $A_{k}$. Hence,

$$
\begin{aligned}
p(x) & =\prod_{i=1}^{n} A_{i}(x)=A_{1}(x) \cdots(f(x)+g(x)) \cdots A_{n}(x) \\
& =A_{1}(x) \cdots f(x) \cdots A_{n}(x)+A_{1}(x) \cdots g(x) \cdots A_{n}(x) .
\end{aligned}
$$

Since $p$ is extremal in $S_{n}$, there exists $\alpha \geqq 0$ and $\beta \geqq 0$ such that $A_{1}(x) \cdots f(x) \cdots A_{n}(x)=\alpha p(x) \quad$ and $\quad A_{1}(x) \cdots g(x) \cdots A_{n}(x)=$ $\beta p(x)$. Let $x \in$ int $E_{n^{2}}^{+}$. Then $p(x)>0$. Also, it can be shown that each $A_{i}(x)>0, f(x)>0$ and $g(x)>0$. Therefore,

$$
\alpha A_{1}(x) \cdots A_{k}(x) \cdots A_{n}(x)=\alpha p(x)=A_{1}(x) \cdots f(x) \cdots A_{n}(x),
$$

which implies that $\alpha A_{k}(x)=f(x)$, for all $x \in$ int $E_{n^{2}}^{+}$. It follows from continuity that $\alpha A_{k}(x)=f(x)$ for all $x \in E_{n^{2}}^{+}$. This is a contradiction. Therefore, $A_{k}$ is an extremal element of $P_{n}^{\prime}$ for each $k$.

In any convex cone, if the sum of two nonzero elements is an extremal element, then the two elements are proportional. Hence, the only possible extremal elements of $S_{n}$ are those elements of the form

$$
p(x)=\prod A_{i}^{l(i)}(x)
$$

where $l(i)$ is a nonnegative integer and $\Sigma l(i)=n$. Moreover, Lemma 1.4 implies that the $A_{i}$ must be extremal elements of $P_{n}^{\prime}$. The Lemma 1.4 and these comments give conditions that are necessary when $p$ is an extremal element in $S_{n}$. These conditions are not sufficient as will be seen in Proposition 1.1.

Attention will now be given to considering the extremal elements of $P_{n}$.

THEOREM 1.2. Let $p$ be defined as in (1.2). Let $k$ be the number of $i$ for which $l(i)>0$. If $k>1$, then $p$ is not an extremal element of $P_{n}$.

Proof. Assume

$$
p(x)=\prod_{i=1}^{k} A_{i}^{l(i)}(x)
$$


where each $l(i)$ is a positive integer, $\sum_{i=1}^{k} l(i)=n$ and the $A_{i}$ are distinct (pairwise nonproportional) extremal elements of $P_{n}^{\prime}$. For each $i \in\{1,2\}$ define

$$
f_{i}(x)=\left\{\begin{array}{cc}
\frac{A_{i}(x)}{A_{1}(x)+A_{2}(x)} p(x), & A_{1}(x)+A_{2}(x)>0 \\
0 & A_{1}(x)+A_{2}(x)=0
\end{array}\right.
$$

It follows easily that $p=f_{1}+f_{2}$. It will now be shown that each $f_{i} \in P_{n}$.

n-Homogeneity: Let $\alpha \geqq 0$ and $x \in E_{n^{2}}^{+}$. If $\alpha=0$, then $A_{1}(\alpha x)=$ $A_{2}(\alpha x)=0$ and hence $f_{i}(\alpha x)=0=\alpha^{n} f_{i}(x)$. Suppose $\alpha>0$. If $0=$ $A_{1}(\alpha x)+A_{2}(\alpha x)=\alpha\left(A_{1}(x)+A_{2}(x)\right)$, then $A_{\mathrm{i}}(x)+A_{2}(x)=0$ and hence $f_{i}(\alpha x)=\alpha^{n} f_{i}(x)$. Suppose $\alpha>0$ and

$$
\alpha\left(A_{1}(x)+A_{2}(x)\right)=A_{1}(\alpha x)+A_{2}(\alpha x)>0,
$$

then $A_{1}(x)+A_{2}(x)>0$. Therefore,

$$
f_{i}(\alpha x)=\frac{A_{1}(\alpha x)}{A_{1}(\alpha x)+A_{2}(\alpha x)} p(\alpha x)=\alpha^{n} \frac{A_{i}(x)}{A_{1}(x)+A_{2}(x)} p(x)=\alpha^{n} f_{i}(x) .
$$

So for all $\alpha \geqq 0$ and $x \in E_{n^{2}}^{+}, f_{i}(\alpha x)=\alpha^{n} f_{i}(x)$.

Superadditivity: Let $x, y \in E_{n^{2}}^{+}$.

Case I. If $A_{1}(x+y)+A_{2}(x+y)=0$, then

$$
0=A_{1}(x+y)+A_{2}(x+y) \geqq A_{1}(x)+A_{1}(y)+A_{2}(x)+A_{2}(y) \geqq 0
$$

which implies that $A_{1}(x)+A_{2}(x)=0$ and $A_{1}(y)+A_{2}(y)=0$. Therefore, $f_{i}(x+y)=f_{i}(x)+f_{i}(y)$.

Case II. Suppose that $A_{1}(x+y)+A_{2}(x+y)>0, A_{1}(x)+A_{2}(x)=$ 0 and $A_{1}(y)+A_{2}(y)=0$. Clearly, $f_{i}(x+y) \geqq f_{i}(x)+f_{i}(y)$.

Case III. Suppose $A_{1}(x+y)+A_{2}(x+y)>0, \quad A_{1}(x)+A_{2}(x)>0$ and $A_{1}(y)+A_{2}(y)=0$. Then

$$
\begin{gathered}
f_{i}(x+y)=\frac{A_{i}(x+y)}{A_{1}(x+y)+A_{2}(x+y)} p(x+y), \\
f_{i}(x)=\frac{A_{i}(x)}{A_{1}(x)+A_{2}(x)} p(x)
\end{gathered}
$$


and $f_{i}(y)=0$. It must be shown that

$$
\frac{A_{i}(x+y)}{A_{1}(x+y)+A_{2}(x+y)} p(x+y) \geqq \frac{A_{i}(x)}{A_{1}(x)+A_{2}(x)} p(x) .
$$

This is true if and only if

$$
\begin{aligned}
& \left(A_{1}(x)+A_{2}(x)\right) A_{i}(x+y) \prod_{j=1}^{k} A_{j}^{l(j)}(x+y) \\
\geqq & \left(A_{1}(x+y)+A_{2}(x+y)\right) A_{i}(x) \prod_{j=1}^{k} A_{j}^{l(j)}(x) .
\end{aligned}
$$

It suffices to show that each term on the right hand side of (1.3) is less than or equal to the corresponding term on the left hand side of (1.3). Now for $m=1$ (or $m=2$ )

$$
\begin{aligned}
A_{m}(x) & A_{i}(x+y) \prod_{j=1}^{k} A_{j}^{l(j)}(x+y) \\
= & A_{m}(x+y) A_{i}(x+y)\left(A_{m}(x) A_{m}^{l(m)-1}(x+y) \cdots A_{k}^{l(k)}(x+y)\right) \\
& \geqq A_{m}(x+y) A_{i}(x) \prod_{j=1}^{k} A_{\jmath}^{l(j)}(x) .
\end{aligned}
$$

It follows that (1.3) is true.

Case IV. Suppose $A_{1}(x+y)+A_{2}(x+y)>0, \quad A_{1}(x)+A_{2}(x)>0$ and $A_{1}(y)+A_{2}(y)>0$. Then

$$
\begin{aligned}
f_{i}(x+y) & =\frac{A_{i}(x+y)}{A_{1}(x+y)+A_{2}(x+y)} p(x+y), \\
f_{i}(x) & =\frac{A_{i}(x)}{A_{1}(x)+A_{2}(x)} p(x),
\end{aligned}
$$

and

$$
f_{i}(y)=\frac{A_{i}(y)}{A_{1}(y)+A_{2}(y)} p(y) .
$$

It must be shown that

$$
\frac{A_{i}(x+y)}{A_{1}(x+y)+A_{2}(x+y)} \prod_{j=1}^{k} A_{j}^{l(j)}(x+y)
$$

$$
\geqq \frac{A_{i}(x)}{A_{1}(x)+A_{2}(x)} \prod_{j=1}^{h} A_{j}^{\ell(j)}(x)+\frac{A_{i}(y)}{A_{1}(y)+A_{2}(y)} \prod_{j=1}^{h} A_{j}^{\ell(j)}(y) .
$$


This will be true if and only if

$$
\begin{aligned}
& A_{i}(x+y)\left(A_{1}(x)+A_{2}(x)\right)\left(A_{1}(y)+A_{2}(y)\right) \prod_{j=1}^{k} A_{j}^{l(j)}(x+y) \\
\geqq & A_{i}(x)\left(A_{1}(y)+A_{2}(y)\right)\left(A_{1}(x+y)+A_{2}(x+y)\right) \prod_{j=1}^{k} A_{j}^{l(j)}(x) \\
+ & A_{i}(y)\left(A_{1}(x)+A_{2}(x)\right)\left(A_{1}(x+y)+A_{2}(x+y)\right) \prod_{j=1}^{k} A_{j}^{l(j)}(y) .
\end{aligned}
$$

Since

$$
\begin{aligned}
& A_{i}(x+y)\left(A_{1}(x)+A_{2}(x)\right)\left(A_{1}(y)+A_{2}(y)\right) \prod_{j=1}^{k} A_{j}^{l(j)}(x+y) \\
& \geqq A_{i}(x)\left(A_{1}(x)+A_{2}(x)\right)\left(A_{1}(y)+A_{2}(y)\right) \prod_{i=1}^{h} A_{j}^{l(j)}(x+y) \\
& +A_{i}(y)\left(A_{1}(x)+A_{2}(x)\right)\left(A_{1}(y)+A_{2}(y)\right) \prod_{j=1}^{k} A_{j}^{l(j)}(x+y),
\end{aligned}
$$

it is sufficient to show that $(\mathrm{g}) \geqq(\mathrm{d})$ and $(\mathrm{h}) \geqq(\mathrm{e})$. An argument similar to the one in Case III shows that each term of $(\mathrm{g})$ or $(\mathrm{h})$ is greater than or equal to the corresponding term of (d) or (e). Thus, (c) $\geqq(d)+(e)$ and hence $(a) \geqq(b)$. Therefore, each $f_{i}$ is superadditive.

Continuity: Let $x \in E_{n^{2}}^{+}$and $\left\{y_{j}\right\} \subset E_{n^{2}}^{+}$such that $y_{j} \rightarrow x$. Suppose $A_{1}(x)+A_{2}(x)>0$, then without loss of generality it may be assumed tha $A_{1}\left(y_{j}\right)+A_{2}\left(y_{j}\right)>0$ for each $j$. In this case

$$
f_{i}\left(y_{j}\right)=\frac{A_{i}\left(y_{j}\right)}{A_{1}\left(y_{j}\right)+A_{2}\left(y_{j}\right)} p\left(y_{j}\right) \rightarrow \frac{A_{i}(x)}{A_{1}(x)+A_{2}(x)} p(x)=f_{i}(x) .
$$

Suppose that $A_{1}(x)+A_{2}(x)=0$, then $p(x)=f_{i}(x)=0$. If there exists $m \in\{1,2\}$ such that $A_{m}\left(y_{j}\right)=0, f_{i}\left(y_{j}\right)=0=f_{i}(x)$. Suppose $A_{m}\left(y_{j}\right)>0$ for $m=1,2$. Since each $A_{m}^{l(m)}\left(y_{j}\right) \rightarrow 0$ and the expression

$$
\frac{A_{i}\left(y_{j}\right)}{A_{1}\left(y_{j}\right)+A_{2}\left(y_{j}\right)}
$$

is obviously bounded by 1 , then $f_{i}\left(y_{j}\right) \rightarrow 0$. Therefore, $f_{i}$ is continuous. Hence, each $f_{i} \in P_{n}$.

It remains to be shown that the functions $f_{i}$ form a nonproportional decomposition of $p$. Suppose $f_{i}(x)=\alpha p(x)$, for all $x \in E_{n^{2}}^{+}$. Let $x \in E_{n^{2}}^{+}$. There exists a sequence $\left\{y_{j}\right\} \subset$ int $E_{n^{2}}^{+}$such that $y_{j} \rightarrow x$. Since $y_{j} \in$ int $E_{n^{2}}^{+}$, then $A_{1}\left(y_{j}\right)>0, A_{2}\left(y_{j}\right)>0$ and $p\left(y_{j}\right)>0$. Hence, 


$$
\alpha p\left(y_{j}\right)=f_{i}\left(y_{j}\right)=\frac{A_{i}\left(y_{j}\right)}{A_{1}\left(y_{j}\right)+A_{2}\left(y_{j}\right)} p\left(y_{j}\right)
$$

which implies that

$$
A_{i}\left(y_{j}\right)=\alpha\left(A_{1}\left(y_{j}\right)+A_{2}\left(y_{j}\right)\right) .
$$

Since $A_{i}\left(y_{j}\right) \rightarrow A_{i}(x)$ and $\alpha\left(A_{1}\left(y_{j}\right)+A_{2}\left(y_{j}\right)\right) \rightarrow \alpha\left(A_{1}(x)+A_{2}(x)\right)$, then $A_{i}(x)=\alpha\left(A_{1}(x)+A_{2}(x)\right)$. Since $A_{1}$ and $A_{2}$ are pairwise nonproportional extremal elements in $P_{n}^{\prime}$, this is a contradiction. Therefore, there does not exists $\alpha \geqq 0$ such that $f_{i}=\alpha p$. Hence, the decomposition is nonproportional, which implies that $p$ is not an extremal element of $P_{n}$.

Two questions immediately arise. First, is $f_{i} \in S_{n}$ ? Secondly, is every extremal element of $P_{n}^{\prime}$ of the form $q_{a}$, where $a \in E_{n^{2}}^{+} \backslash\{0\}$ ? If both answers are affirmative, then every extremal element of $S_{n}$ is of the form $p_{a}$, where $a \in E_{n^{2}}^{+} \backslash 0$. It is entirely possible that the functions $f_{i}$ do not belong to $S_{n}$.

The following is an example of a subcone of $P_{n}$ that has as extremal elements some functions that are not extremal in $P_{n}$.

Example 1.1. Let $Q_{n}$ be the set of all $p: E_{n^{2}}^{+} \rightarrow E_{1}^{+}$such that

$$
p(x)=\sum_{i 1, \cdots, i n=1}^{n^{2}} \alpha_{i 1, \cdots, i n} x_{i 1} \cdots x_{i n}
$$

where $i 1 \leqq \cdots \leqq$ in, $\alpha_{i 1, \cdots, i n} \geqq 0$ and $x=\left(x_{1}, \cdots, x_{n^{2}}\right)$. Thus, $Q_{n}$ is the set of nonnegative superadditive $n$-forms. Clearly, $Q_{n}$ is a subcone of $S_{n} \subset P_{n}$. Therefore, the functions $p_{1}, \cdots, p_{n^{2}}$ are extremal elements of $Q_{n}$. However, these are not all of the extremal elements of $Q_{n}$. In fact without much difficulty it can be shown that the extremal elements of $Q_{n}$ are the positive scalar multiples of functions of the form

$$
p(x)=x_{k 1} \cdots x_{k n},
$$

where $k j \in\left\{1, \cdots, n^{2}\right\}$, for $j=1, \cdots, n$ and $k 1 \leqq \cdots \leqq k n$.

Now for every $x=\left(x_{1}, \cdots, x_{n^{2}}\right) \in E_{n^{2}}^{+}$define $p(x)$ as

$$
p(x)=x_{1}^{l(1)} \cdots x_{n^{2}}^{l\left(n^{2}\right)},
$$

where $(i)$ is a nonnegative integer and $\sum_{i=1}^{n^{2}} l(i)=n$. Notice that $l(i)>0$ for at most $n$ values of $i=1, \cdots, n^{2}$. Clearly, $p \in Q_{n}$. In fact the preceding example shows that $p$ is an extremal element of $Q_{n}$. If $k$ is the number of $i \in\left\{1, \cdots n^{2}\right\}$ for which $l(i)>0$ and $k>1$, Theorem 1.2 
says that $p$ is not an extremal element of $P_{n}$. The following proposition shows that $p$ is not an extremal element of $S_{n}$.

Proposition 1.1. Let $p$ be defined as in (1.4). If $k>1$, then $p$ is not an extremal element of $S_{n}$.

Proof. Without loss of generality assume

$$
p(x)=x_{1}^{l(1)} x_{2}^{l(2)} \cdots x_{k}^{l(k)}
$$

where each $l(k)>0$. As seen in the proof of Theorem 1.2, $p=f_{1}+f_{2}$ where

$$
f_{i}(x)=\left\{\begin{array}{cr}
\frac{x_{i}}{x_{1}+x_{2}} x_{1}^{l(1)} \cdots x_{k}^{l(k)}, & x_{1}+x_{2}>0 \\
0 \quad & x_{1}+x_{2}=0 .
\end{array}\right.
$$

Consider $f_{1}$. Notice that

$$
f_{1}(x)\left\{\begin{array}{cr}
\frac{x_{1} x_{2}}{x_{1}+x_{2}} x_{1}^{l(1)} x_{2}^{l(2)-1} \cdots x_{k}^{l(k)}, & x_{1}+x_{2}>0 \\
0 \quad, & x_{1}+x_{2}=0 .
\end{array}\right.
$$

Let

$$
g(x)=\left\{\begin{array}{cc}
\frac{x_{1} x_{2}}{x_{1}+x_{2}}, & x_{1}+x_{2}>0 \\
0, & x_{1}+x_{2}=0
\end{array}\right.
$$

Then $f_{1}(x)=g(x) x_{1}^{l(1)} x_{2}^{l(2)-1} \cdots x_{k}^{l(k)}$. Since the objective is to show that $f_{1} \in S_{n}$, it remains to be shown that $g \in P_{n}^{\prime}$. As in Theorem $1.2 g$ is continuous and homogeneous of degree 1 . To show supperadditively let $x, y \in E_{n^{2}}^{+}$. If $x_{1}+x_{2}=0$ or $y_{1}+y_{2}=0$, then it follows readily that $g(x+y)=g(x)+g(y)$. Suppose $x_{1}+x_{2}>0$ and $y_{1}+y_{2}>0$. In this case it must be shown that

$$
\frac{\left(x_{1}+y_{1}\right)\left(x_{2}+y_{2}\right)}{x_{1}+y_{1}+x_{2}+y_{2}} \geqq \frac{x_{1} x_{2}}{x_{1}+x_{2}}+\frac{y_{1} y_{2}}{y_{1}+y_{2}},
$$

which is equivalent to proving that

$$
\begin{aligned}
\left(x_{1}+y_{1}\right)\left(x_{2}+y_{2}\right)\left(x_{1}+x_{2}\right)\left(y_{1}+y_{2}\right)- & {\left[\left(x_{1} x_{2}\right)\left(x_{1}+y_{1}+x_{2}+y_{2}\right)\left(y_{1}+y_{2}\right)\right.} \\
+ & \left.y_{1} y_{2}\left(x_{1}+y_{1}+x_{2}+y_{2}\right)\left(x_{1}+x_{2}\right)\right] \geqq 0 .
\end{aligned}
$$

By direct calculation the left hand side of the above inequality is equal to $\left(x_{1} y_{2}-x_{2} y_{1}\right)^{2}$. The computation is tedious but straightforward. 
Hence, $g$ is superadditive and $f_{1} \in S_{n}$. Likewise, $f_{2} \in S_{n}$. Hence, $p$ is not an extremal element of $S_{n}$.

Actually, it can be shown that if $p$ is defined as in (1.2) and if at least two of the $A_{i}$ are additive, then $p$ is not an extremal element of $S_{n}$.

\section{REFERENCES}

1. G. Choquet, Theory of capacities, Annales de l'Institut, 5 (1953 and 1954), 131-296.

2. M. Marcus and M. Newman, The permanent as an inner product, Bull. Amer. Math. Soc., 67 (1961), 223-224.

3. M. Marcus and M. Newman, On the minimum of the permanent of a doubly stochastic matrix, Duke Math. J., 26 (1959), 61-72.

4. E. K. McLachlan, Extremal elements of the convex cone of seminorms, Pacific J. Math. 13 (1963), 1335-1342.

5. - Extremal elements of the convex cone $B_{n}$ of functions, Pacific J. Math., 14 (1964), 987-993.

6. - Extremal elements of a convex cone of subadditive functions, Proc. Amer. Math. Soc., 12 (1961), 77-83.

7. R. M. Rakestraw, Extremal elements of the convex cone $A_{n}$ of functions, Pacific J. Math. 34 (1970), 491-500.

8. R. T. Rockafellar, Monotone processes of convex and concave type, Memoirs of the Amer. Math. Soc., 77 (1967), 10.

9. O. S. Rothaus, Study of the permanent conjecture and some generalizations, Bull. Amer. Math. Soc., 18 (1972), 749-751.

Received October 15, 1973 and in revised form May 16, 1974. This paper is part of the author's doctoral thesis which was directed by Professor E. K. McLachlan. The author wishes to express his gratitude to Professor McLachlan and also to acknowledge the many useful suggestions made by the referee.

Oklahoma State University

AND

UNIVERSITY OF SOUTHERN MISSISSIPPI 




\section{Pacific Journal of Mathematics}

\section{Vol. 56, No. $1 \quad$ November, 1975}

Shimshon A. Amitsur, Central embeddings in semi-simple rings .......... 1

David Marion Arnold and Charles Estep Murley, Abelian groups, A, such

that $\operatorname{Hom}(A,---)$ preserves direct sums of copies of $A \ldots \ldots \ldots .$.

Martin Bartelt, An integral representation for strictly continuous linear

operators ................................... 21

Richard G. Burton, Fractional elements in multiplicative lattices......... 35

James Alan Cochran, Growth estimates for the singular values of

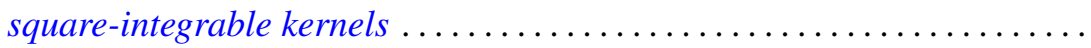

C. Martin Edwards and Peter John Stacey, On group algebras of central

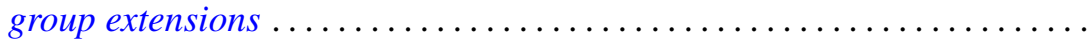

Peter Fletcher and Pei Liu, Topologies compatible with homeomorphism

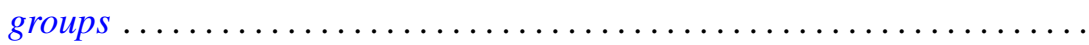

George Gasper, Jr., Products of terminating ${ }_{3} F_{2}(1)$ series ............ 87

Leon Gerber, The orthocentric simplex as an extreme simplex ............

Burrell Washington Helton, A product integral solution of a Riccati

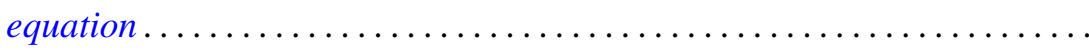

Melvyn W. Jeter, On the extremal elements of the convex cone of

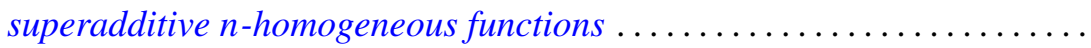

R. H. Johnson, Simple separable graphs .

Margaret Humm Kleinfeld, More on a generalization of commutative and

alternative rings. . .

A. Y. W. Lau, The boundary of a semilattice on an $n$-cell.

Robert F. Lax, The local rigidity of the moduli scheme for curves ...

Glenn Richard Luecke, A note on quasidiagonal and quasitriangular

operators .

Paul Milnes, On the extension of continuous and almost periodic functions

Hidegoro Nakano and Kazumi Nakano, Connector theory.

James Michael Osterburg, Completely outer Galois theory of perfect rings ..................................

Lavon Barry Page, Compact Hankel operators and the F. and M. Riesz

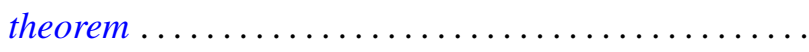

Joseph E. Quinn, Intermediate Riesz spaces..................... 225

Shlomo Vinner, Model-completeness in a first order language with a generalized quantifier.

Jorge Viola-Prioli, On absolutely torsion-free rings ..........

Philip William Walker, A note on differential equations with all solutions of integrable-square............................

Stephen Jeffrey Willson, Equivariant maps between representation 